\title{
Apical Aneurysm and Left Ventricular Hypertrophy
}

\author{
Helena Nogueira Soufen, Clovis de Carvalho Frimm, Luiz Alberto Benvenuti, Charles Mady
}

São Paulo, SP - Brazil

\begin{abstract}
A 59-year-old woman presented with an embolic transient ischemic attack and a history of controlled hypertension for 16 years. Both echocardiogram and MRI showed severe biventricular hypertrophy and an apical aneurysm with a thrombus. The occurrence of an apical aneurysm in the presence of cardiac hypertrophy is a rare finding and has been described in patients with hypertrophic cardiomyopathy. However, it has not been reported in patients with systemic arterial hypertension. In this patient the lack of a relationship between the severity of the hypertrophy and the levels of blood pressure, together with the presence of histologic disorganization of myocardial cardiac muscle cells by endomyocardial biopsy suggested the diagnosis of hypertrophic cardiomyopathy.
\end{abstract}

Left ventricular hypertrophy represents an adaptation of the heart to the increased afterload that occurs in systemic hypertension. However, in some cases, the presence and the degree of hypertrophy do not correlate with the severity of hypertension, which has already been described in the elderly and in patients with pheochromocitoma ${ }^{1}$. Other factors, such as the level of the arterial blood pressure, plasmatic renin activity and circulating catecholamines seem to relate to the degree of hypertrophy. Because hypertension is a disease with a high prevalence in the population, an association with hypertrophic cardiomyopathy in some patients is also a possibility ${ }^{2}$. This is the report of a patient with moderate hypertension and evidence of severe hypertrophy associated with the presence of an apical aneurysm of the left ventricle, which is a complication rarely seen in hypertrophic cardiomyopathy ${ }^{3}$ that has not been described in cases of isolated hypertension.

\section{Case Report}

The patient is a 59-year-old woman with a history of smoking cigarettes and hypertension since 1982 when she started regular control of her blood pressure as an outpatient. She reported no family history of heart disease or sud-

Instituto do Coração do Hospital das Clínicas - FMUSP

Mailing address: Helena Nogueira Soufen - Incor - Unidade Clínica de Cardiopatias Gerais - Av. Dr. Enéas C. Aguiar, 44 - 05403-900 - São Paulo, SP - Brasil - E-mail: cardebora@incor.usp.br den death. On physical examination her blood pressure was $160 / 95 \mathrm{mmHg}$ and her heart rate was 76bpm. The apical thrust was palpable in the $5^{\text {th }}$ intercostal space of the left midclavicular line and had an increased impulse. On cardiac auscultation, heart sounds were rhythmic and had normal intensity, and a ++/4+ ejective systolic murmur could be heard at the base of the precordium with no irradiation. Carotid pulse was normal on palpation. Exams to diagnose secondary causes of systemic hypertension were negative, as were serological exams for Chaga's disease.

The patient was asymptomatic, with controlled levels of blood pressure on atenolol and chlortalidone until mid1997. She then began to experience fatigue and increasing precordial pain with exercise. Eletrocardiogram and echocardiogram showed signs suggestive of left ventricular hypertrophy.

In August 1998, the patient had an ictal episode of aphasia of expression and left hemiparesis that lasted for 30 minutes and showed complete regression. Her clinical presentation did not suggest hypertensive crisis and the diagnosis of probable thromboembolic transient ischemic attack was made. Examinations to detect the source of the emboli were performed. Severe hypertrophy of the left ventricle was diagnosed by echocardiography, where a $21 \mathrm{~mm}$ septum and a $16 \mathrm{~mm}$ posterior wall thickness were observed. An intraventricular gradient of $120 \mathrm{mmHg}$ was detected by Doppler.

MRI (figure 1) confirmed the echocardiographic finding of severe hypertrophy associated with a mesoventricular obstruction of the left ventricle and the presence of an apical aneurysm with a thrombus. Hypertrophy of the right ventricle was also present. Coronary angiography was indicated and the coronary arteries were shown to be normal. Endomyocardial biopsy of the right ventricular was then indicated due to the unknown cause of the cardiac disease and to its severity. Histology of the biopsy fragments by optical microscopy ruled out storage diseases and confirmed the presence of hypertrophy with disorganization of the myocytes (fig. 2). Disorganization of the myofibrilla also existed as shown by electronic microscopy (fig. 3).

Oral anticoagulation with warfarin was initiated and verapamil was associated with complete regression of the symptoms of the encephalic ischemia. Although cardiac symptoms improved, allowing the patient to resume her routine professional activities, they did not show complete regression. 


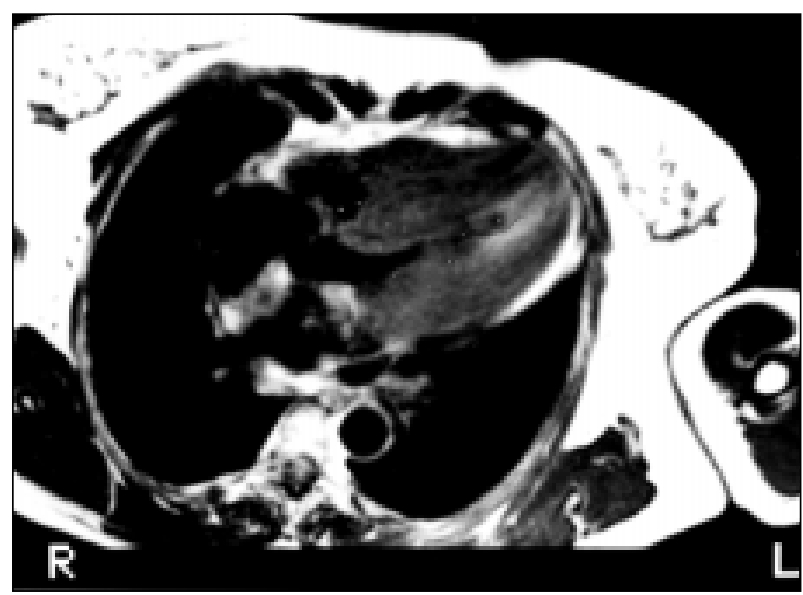

Fig. 1 - MRI picture of the four chamber view in systole showing severe biventricular hypertrophy, apical aneurysm of the left ventricle and an intracardiac thrombus.

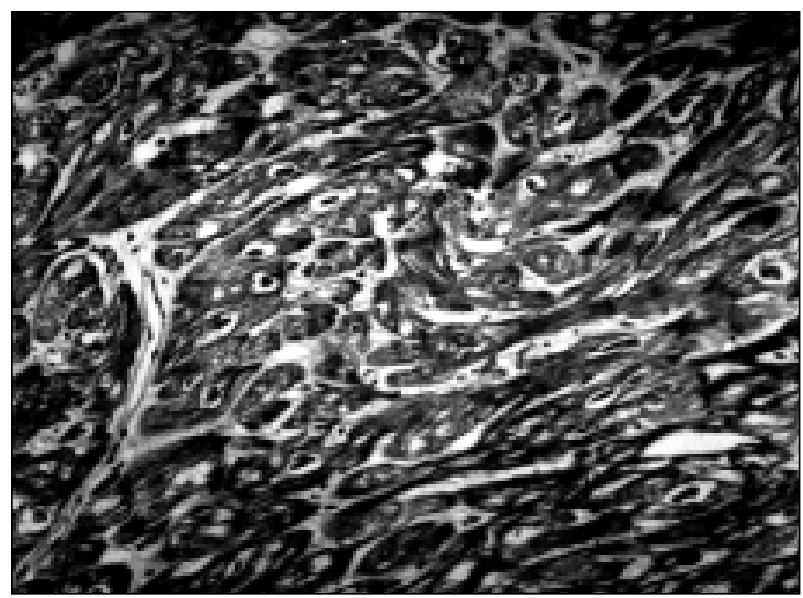

Fig. 2-Photomicrography of myocadial tissue obtained from biopsy of the right ventricle demonstrating hypertrophy and mild disorganization of the myocytes. HE, 250x.

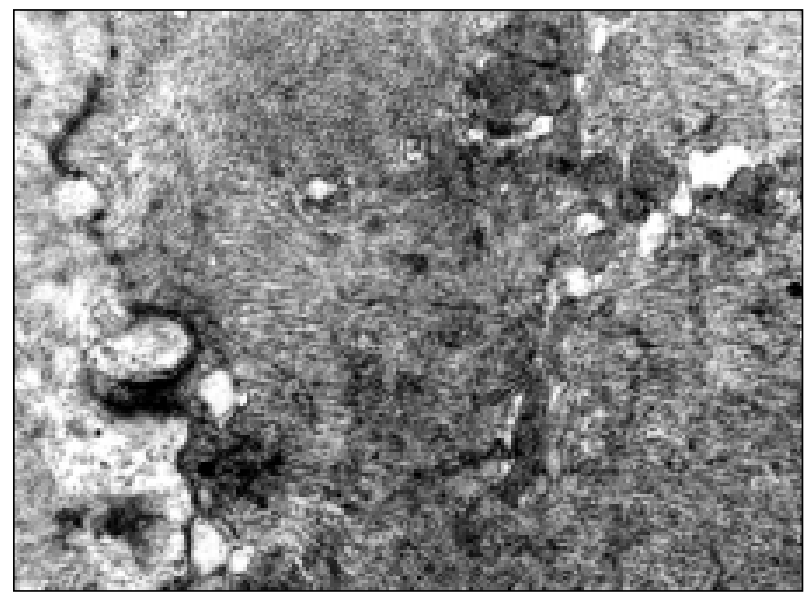

Fig. 3 - Electronic photomicrography of a myocardial fragment showing myocyte with disorganization of the myofibrilla. 22000x.

\section{Discussion}

This is the report of a patient with the diagnosis of ar- terial hypertension who, in spite of adequate clinical control, showed progressive hypertrophy of the left ventricle and developed and apical aneurysm with a thrombus, which was complicated by a transient ischemic attack. MRI confirmed the echocardiographic findings of asymmetric hypertrophy with mesoventricular obstruction and the presence of an apical aneurysm with a thrombus. Its contribution to the ecocardiographic diagnosis consisted in the detection of the right ventricular hypertrophy.

The occurrence of a left ventricular aneurysm in patients with cardiac hypertrophy, in the absence of coronary artery disease, has been described in patients with hypertrophic cardiomyopathy ${ }^{3}$. As far as we know, this complication had not been described in patients with hypertrophy secondary to arterial hypertension.

In hypertrophic cardiomyopathy, when mesoventricular obstruction exists, the apex of the left ventricle is subjected to a chronic increase of systolic stress, which predisposes to repeated episodes of ischemia and progressive necrosis. With decreased apical contractility and increased wall stress, a vicious cycle is established leading to the formation of an aneurysm ${ }^{3}$. It seems logical that the reduction in coronary artery flow reserve that occurs in hypertrophic cardiomyopathy can also contribute to aneurysm formation ${ }^{3}$.

Because arterial hypertension is a highly prevalent disease in the population, the association of hypertension with other diseases is possible ${ }^{4}$. In Brazil de presence of an apical aneurysm is always suggestive of Chagas's disease. However, two systematically negative serological exams specific for Chagas's ruled out this disease. Echocardiographic findings suggestive of hypertrophic cardiomyopathy have been described in patients with arterial hypertension and the degree of hypertrophy sometimes does not correlate with the severity of the hypertension, as seen in young patients with hypertension, in the elderly and in some cases of secondary hypertension. In these cases, the treatment of hypertension with the regression of the cardiac hypertrophy can help in defining the diagnosis. We have seen complete regression of severe asymmetrical cardiac hypertrophy after treatment of pheochromocytoma ${ }^{1}$. A family history can help in the diagnosis of hypertrophic cardiomyopathy but its absence does not rule out the disease. However, not much has been published in the literature to help differentiate hypertrophic cardiomyopathy from the hypertrophy that occurs in arterial hypertension. Some authors have compared clinical signs and parameters by echocardiography, ventriculography and endomyocardialfibrosis between patients with hypertension and hypertrophic cardiomyopathy ${ }^{4-6}$.

Only some differences in parameters of diastolic function, such as a shorter isovolumic relaxation time in hypertension, have been found. However, none of the studied parameters differentiated between the two entities. The results obtained from endomyocardial biopsy seem to help in distinguishing between the two ${ }^{5,6}$. In fact, although 
cardiac muscle-cell disorganization is not pathognomonic in hypertrophic cardiomyopathy, a previous study has shown it to be present in $86 \%$ of the cases of this disease and in only $14 \%$ of the cases of arterial hypertension ${ }^{5}$. Other histologic findings, such as myocardial fibrosis and the diameter of the myocytes, are similar in the two entities ${ }^{6}$. Although the quantity of fibrosis is similar, the space distribution of collagen fibers is not; it is plexiform in hypertrophic cardiomyopathy, indicating the disorganization of the myocytes, while in arterial hypertension, the pattern of distribution of the collagen fibers is normal ${ }^{6}$.

Taking these facts into consideration, clinical data in this patient suggest an association of the two diseases: systemic arterial hypertension and hypertrophic cardiomyopathy. This hypothesis was raised due to the occur- rence of a rare complication of hypertrophic cardiomyopathy: the formation of an apical aneurysm.

Therefore, this case shows that the increase in the left ventricle that occurs in the presence of hypertension may not be related to an increase in afterload and may represent a rare event due to the presence of a concomitant disease. In the present case, the diagnosis of hypertrophic cardiomyopathy was made because the severity of the hypertrophy was not proportional to the level of hypertension, it did not regress with adequate control of blood pressure, and it was associated with a complication that has been reported in hypertrophic cardiomyopathy. Currently histologic studies of the myocardium, although not definitely diagnosis, help in establishing the diagnosis, especially because it rules out inflammatory and storage diseases.

\section{References}

1. Lopes HF, Silva HB, Frimm CC, Bortolotto LA, Belotti G, Pileggi F. Falso diagnóstico de miocardiopatia hipertrófica em feocromocitoma. Arq Bras Cardiol 1995; 65: 167-79.

2. Lewis JF, Maron BJ. Diversity of patterns of hypertrophy in patients with systemic hypertension and marked left ventricular wall thickening. Am J Cardiol 1990; 65: 874-81.

3. Matsuura H, Yasuda T. Hypertrophic cardiomyopathy complicated with ventricular aneurysm and myocardial necrosis. J Nucl Med 1993; 34: 2227-35.

4. Karan R, Lever HM, Healy BP. Hypertensive hypertrophic cardiomyopathy or hypertrophic cardiomyopathy with hypertension: a study of 78 patients. J Am Coll Cardiol 1989; 13: 580-4.

5. Shimizu M, Sugihara N, Shimizu K, et al. Asymmetrical septal hypertrophy in patients with hypertension: a type of hypertensive left ventricular hypertrophy or hypertrophic cardiomyopathy combined with hypertension. Clin Cardiol 1993; 16: 41-6.

6. Sugihara N, Genda A, Shimizu M, et al. Quantitation of myocardial fibrosis and its relation to function in essential hypertension and hypertrophic cardiomyopathy. Clin Cardiol 1978; 11: 771-8. 